\title{
Umbilical Artery Resistance Index in the Surveillance of Pregnancies at Risk of Fetal-Maternal Hemodynamic Disorders in Lomé (Togo)
}

\author{
Sonhaye Lantam1, Kolou Bérésa', Boube Abarchi Habibou ${ }^{*}$, Bah Ousmane Aminata1, \\ Amadou Abdoulatif', Gbande Pihou1, Assih Kouméabalo1, Tchaou Mazamaesso², \\ Ntimon Bidamin ${ }^{3}$, Adjenou Komlanvi ${ }^{1}$
}

\author{
${ }^{1}$ Radiology Department of Campus Teaching Hospital of Lomé, Lomé, Togo \\ ${ }^{2}$ Radiology Department of Sylvanus Olympio Teaching Hospital of Lomé, Lomé, Togo \\ ${ }^{3}$ Radiology Department of Kara Teaching Hospital of Kara, Lomé, Togo \\ Email: ^habibouabarchi@gmail.com
}

How to cite this paper: Lantam, S., Bérésa, K., Habibou, B.A., Aminata, B.O., Abdoulatif, A., Pihou, G., Kouméabalo, A., Mazamaesso, T., Bidamin, N. and Komlanvi, A. (2018) Umbilical Artery Resistance Index in the Surveillance of Pregnancies at Risk of Fetal-Maternal Hemodynamic Disorders in Lomé (Togo). Open Journal of Radiology, 8, 159-166.

https://doi.org/10.4236/ojrad.2018.83018

Received: May 30, 2018

Accepted: August 26, 2018

Published: August 29, 2018

Copyright $\odot 2018$ by authors and Scientific Research Publishing Inc. This work is licensed under the Creative Commons Attribution International License (CC BY 4.0).

http://creativecommons.org/licenses/by/4.0/

\section{(c) (i) Open Access}

\begin{abstract}
Objective: Evaluate the role of umbilical artery Doppler ultrasound in the surveillance of pregnancies at risk of vascular disorders in Lomé. Study Method: The study was a cross-sectional analytical study carried out in the department of radiology of Campus teaching hospital over a period of 6 months. This study was based on the measurement of the resistance index of the umbilical artery in pregnant women presenting vascular risk and other pregnant women without vascular risk. The correlation between the pathological index and the at-risk pregnancies was assessed by the Odds Ratio as well as the correlation between the resistance index and the Apgar score at birth. Results: The resistance index was measured in 209 at-risk pregnant women and in 425 pregnant women without vascular risk. The average age of pregnant women was 26.31 years for "the at-risk pregnant" versus 25.38 years for the "pregnant-witnesses". The association between the pathological resistance index (RI) and the gestational pathologies studied, had been positive and significant with an odds ratio of 1.57 for a $95 \%$ confidence interval of [1.07 - 2.20]. A pathological RI is a risk factor for the occurrence of a pathological Apgar score at birth because this association was positive and significant for "pregnant-cases" as for "pregnant-witnesses". Conclusion: Measuring the index of resistance is not a common practice in our communities. However, it could be an important tool in the surveillance of at-risk pregnancies for diseases such as malaria, pre-eclampsia, and maternal anemia.
\end{abstract}




\section{Keywords}

Umbilical Doppler, Pre-Eclampsia, Malaria, Lomé

\section{Introduction}

Pregnancy is defined as a risky pregnancy when it presents pathological and physiological situations or antecedents situations with a particular risk for the mother, the fetus, and the newborn [1]. These situations are reflected in the mother by maternal age, pre-eclampsia, eclampsia, uterine rupture, malaria, and anemia. In the fetus they result in fetal distress, infection, in-utero death, prematurity, and malformations. They are an important health problem because of their impact on the maternal and fetal health. Their occurrence and complications are one of the leading causes of maternal death. Ten (10) to twenty (20) percent of pregnancies are considered risky and it is estimated that in general $50 \%$ of perinatal mortality and morbidity are related to these at-risk pregnancies [2].

Doppler examination and more particularly measurement of umbilical artery indices provide information on placental dysfunction. This measurement monitors changes in fetal hemodynamics and the more the fetus is reached, the more arterial and venous blood flows are disrupted [3] [4]. Studies have also been conducted on the contribution of Doppler ultrasound in assessing fetal well-being during high-risk pregnancies in obstetric care and fetal outcomes [5] [6].

In sub-Saharan Africa, despite progress in the management of women with risky pregnancies, umbilical artery Doppler velocimetry remains uncommon. Thus we chose to carry out this work with the general objective of determining the interest of the index of vascular resistance in Umbilical artery Doppler ultrasound during the surveillance of at-risk pregnancies in Lomé.

\section{Materials and Study Method}

This was a cross-sectional analytical study carried out in the radiology department of the Lomé University Hospital Center from 1 February 2013 to 31 July 2014. This study focused on the measurement of the umbilical artery resistance index (UARI) in pregnant women with high-risk pregnancies and pregnant women with no vascular risk.

We included pregnant women bearing a mono fetal pregnancy in development with at least 28 weeks of amenorrhea (WA) at obstetrical ultrasound, whose date of the last menstrual period (LMP) is known with precision and who achieved the first trimester ultrasound scan before 12 weeks of amenorrhea (WA). We considered "pregnant-at-risk" a pregnant woman who had malaria on pregnancy (positive test or positive plasmodium falciparum test in the second and/or third trimester), or anemia on pregnancy (hemoglobin concentration 
which was strictly less than $11 \mathrm{~g} / \mathrm{dL}$ in the second and/or third trimester), or pre-eclampsia in pregnancy (any pregnancy during which, starting from 20 weeks, pregnant women presented a blood pressure higher than $130 \mathrm{mmHg}$ systolic and $85 \mathrm{mmHg}$ diastolic, associated with positive qualitative proteinuria).

We included as pregnant witnesses the pregnant women whose pregnancy was not at risk for the aforementioned pathologies and who did not present any particular pathology.

We excluded the pregnant women who had more than one of the three pathologies at risk of hemodynamic disorders, and the pregnant women with mechanical abnormalities of the umbilical cord.

We used as equipment a General Electric LOGIPQ P5 branded ultrasound system with multifrequency probes, equipped with the color Doppler module, pulsed Doppler with its different modes: duplex, triplex, and alternate.

All exams were performed by the same operator, a radiologist with at least five years of experience in Doppler.

We set the filter between 50 and $100 \mathrm{~Hz}$. We manually set the PRF according to the quality of the resulting plot and the observed speeds. The firing angle was between 30 and 60. We placed the Doppler gate as far as possible in the center of the vessel to be studied.

The examination began by locating the insertion of the umbilical cord at the level of placenta and then we used the color Doppler mode to find one of the umbilical arteries. The pulsed Doppler mode was used to obtain the path corresponding to the selected artery, with at least three consecutive pulses that we examined and on which we measured velocimetry parameters (the resistance index).

We considered that the resistance index was normal when it was $0.78(+/-0.05$ DS) between 28 and $30 \mathrm{WA} ; 0.66(+/-0.05$ DS) between 31 and 33 AS; 0.62 $(+/-0.05$ DS) between 34 and 37 WA; 0.57 (+/ -0.06 DS) between 38 and 41 WA.

The weight of the newborn at birth was normal when it was between 2500 and 3500 grams; Apgar's score was normal when it was above 7 in the first minute.

The correlation between the resistance index and the pathologies was evaluated using the Odds Ratio (OR).

\section{Results}

We performed Doppler examination of the umbilical artery in a total of 634 pregnant women including 209 "pregnant-at-risk" and 425 "pregnant-witness".

The mean age of "pregnant-at-risk" was 26.31 years with extremes of 17 to 42 years, while among gestating-controls the average age was 25.38 with the same extremes.

Of the 209 "at-risk pregnant women", 125 (59.8\%) had anemia; 31 (14.8\%) had malaria, and 53 (25.4\%) had pre-eclampsia.

Resistance Index (RI) was normal in 117 (55.98\%) "at-risk pregnant" and 383 (90.12\%) "pregnant-controls". RI was pathological in 92 (44.02\%) "at-risk preg- 
nant" and $42(09.88 \%)$ "gestating-controls" (Figure 1). The calculation of the odds ratio made possible the evaluation of the association between a pathological resistance index and pregnant women with maternal vascular diseases (pre-eclampsia, malaria, and anemia). The odds ratio is 1.57 for a $95 \%$ confidence interval (IC) of [1.07 - 2.20].

IR was predominantly normal in pregnant women with pre-eclampsia and in those with malaria and anemia (Table 1). In patients who had pre-eclampsia during pregnancy, the evaluation of the association between pathological RI and pre-eclampsia yielded a OR of 16.32 for CI [8.58 - 30.87].

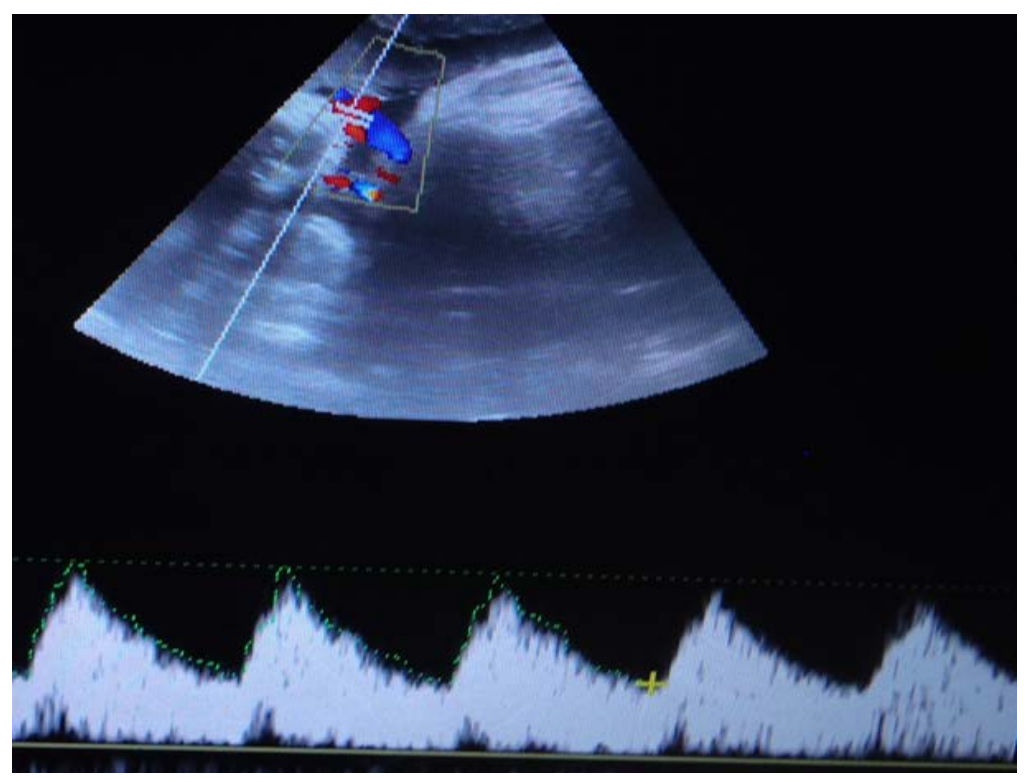

(a)

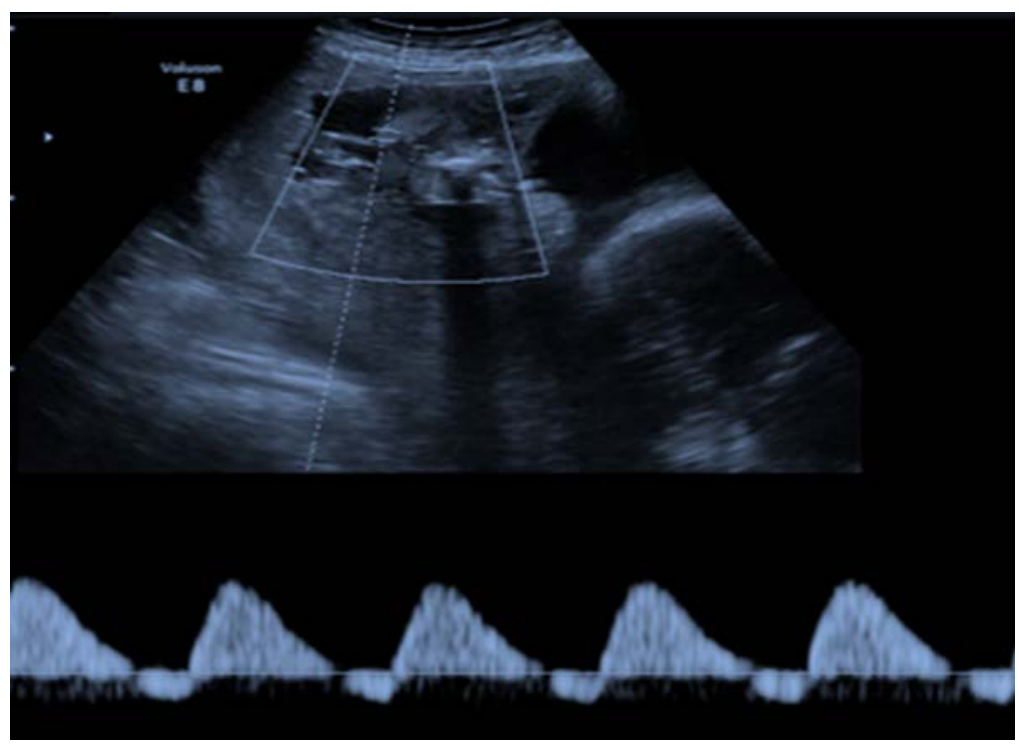

(b)

Figure 1. Umbilical artery pulsed Doppler waveforms: (a) in a 30-year-old pregnant witness at 34 weeks of amenorrhea; (b) in a 24-year-old pregnant woman at 35 weeks of amenorrhea who had preeclampsia. 
Table 1. Classification of the pregnant women at-risk according to the maternal pathologies and the RI.

\begin{tabular}{ccccccc}
\hline & Pre-eclampsia & $\%$ & Anemia & $\%$ & Malaria & $\%$ \\
\hline Pathological RI & 34 & 64.15 & 50 & 40.00 & 08 & 25.81 \\
Normal RI & 19 & 35.85 & 75 & 60.00 & 23 & 74.19 \\
Total & 53 & 100 & 125 & 100 & 31 & 100 \\
\hline
\end{tabular}

RI: Resistance Index.

The Apgar score was pathological in 9 (4.3\%) "at-risk pregnant" and 15 (3.5\%) "pregnant-controls". The evaluation of the association between IR and Apgar score in "at-risk pregnant women" gave an OR of 4.73 for a CI [1.35 90.01]. However, the evaluation of the association between IR and Apgar score in the "pregnant-controls" gave an OR of 6.92 for a CI [2.31 - 20.49].

\section{Discussion}

The average age of "at-risk pregnant women" was 26.3 years with extremes of 17 to 42 years, while in pregnant women-controls the average age was 25.4 with the same extremes. Our results are consistent with those of Da Silva [3] in Brazil who found an average maternal age of 29.1 years in the women-cases and 26.1 in the women-controls. Similarly, Anshul [7] in India, Valea [8] in Burkina, and Wilson [9] in Ghana had respectively an average age of 27.2 years, 24.4 years, and 28.8 years with extremes of 18 to 44 years for the latter. This result is explained by the fact that this age corresponds to the age of a high fertility.

The RI in our study was normal in 117 (55.78\%) "at-risk pregnant women" and in 383 (90.12\%) "pregnant-controls women". The RI was pathological in 92 (44.02\%) "at-risk pregnant women" and in 42 (09.88\%) "pregnant women-controls". The calculation of the odds ratio made possible the evaluation of the association between a pathological resistance index and pregnant women with maternal vascular diseases (preeclampsia, malaria, and anemia). The odds ratio is 1.57 for a $95 \%$ confidence interval of [1.07 - 2.20]. As the OR is greater than 1 , the association is positive and significant. This means that the risk of an abnormal resistance index is higher in pregnant women (suffering from anemia, pre-eclampsia, and malaria).

Studies [10] have noted an association between the pathological resistance index and at-risk pregnancies. They concluded that umbilical artery Doppler in a high-risk population appeared to improve a number of outcomes in obstetric care and looked promising in reducing the number of perinatal deaths.

The association between pathological RI and pre-eclampsia was positive and significant $(\mathrm{OR}=16.32$ for a $\mathrm{CI}$ of 8.58 - 30.87). Therefore, the occurrence of pre-eclampsia is a factor of risk for obtaining a pathological RI of the umbilical artery. Lopez-Mendez [11] in Mexico found a positive association between RI abnormalities and pre-eclampsia with an odds ratio of 30.63 for a $95 \%$ confidence interval between 1.47 and 639.71. These results are relatively similar to ours, with in both cases a high value of the OR but a much smaller confidence 
interval in our series.

Doppler ultrasound is generally considered as a valid method to assessing fetal well-being in high-risk women, particularly for preeclampsia [12] [13].

The association between the RI and malaria was also positive and significant (OR of 3.17 for a CI of 1.46 - 6.82). The appearance of an index of pathological resistance to umbilical Doppler is more frequent in pregnant women with malaria than in those without malaria. Studies in Kenya [14] [15] and in Papua New Guinea [16] also found a statistically significant association between elevation of UARI and malaria in pregnant women. Similarly, Arbeille [17] [18] noted that symptomatic malaria at the beginning of the third trimester was associated with an increase in UARI in French Guyana.

Regarding the pregnant women with anemia during pregnancy, the evaluation of the association between the RI was positive and significant anemia (OR 9.08 for a CI of 3.74 - 9.77); which makes anemia be a factor of risk for umbilical Doppler RI during pregnancy. Studies [19] [20] [21] have noted that when Doppler velocimetry abnormalities are accompanied by maternal anemia, the risk of adverse pregnancy outcomes is increased.

By contrast, Carles [22] noted that a severe maternal anemia (hemoglobin-6 $\mathrm{g} / \mathrm{dL}$ ) triggered fetal brain vasodilatation, but did not alter the resistance of the umbilical and uterine arteries.

The Apgar score was pathological in 9 (4.3\%) "at-risk pregnant women" and 15 (3.5\%) "pregnant women-controls". The association between pathological RI and Apgar score in "at-risk pregnant women" was positive and significant (OR = 4.73 for a CI of 1.35 - 90.01). A pathological RI of the umbilical artery in "at-risk pregnant" is significantly related to an abnormal Apgar score at birth. Similarly, studies [23] [24] have shown that the association of pathological pregnancy and the measurement of the RI in at-risk pregnant women can then be useful in predicting the adverse outcome of pregnancy and therefore can be a surveillance tool for pregnant women at risk.

\section{Conclusion}

Pregnancy at risk is common in our communities. The use of umbilical artery Doppler in pregnancy is not a common practice. However, the measurement of the resistance index of the umbilical artery in pregnancy at risk (preeclampsia, anemia, and malaria) might be a tool to predicting adverse outcomes of pregnancy.

\section{Conflicts of Interest}

The authors declare no conflicts of interest regarding the publication of this paper.

\section{References}

[1] Chaman, R., Holakouie, N.K., Golestan, B., Nabarizadeh, H. and Yunesian, M. (2009) Neonatal Mortality Risk Factors in a Rural Part of Iran: A Nested Case Con- 
trol Study. Iranian Journal of Public Health, 38, 48-52.

[2] Behrman, R.E., Kliegman, R.M. and Jenson, H.B. (2004) Nelson Textbook of Pediatrics. Vol. 1, 17th Edition, Saunders Co., Philadelphia, PA.

[3] da Silva, F.C., Moreira de Sá, R.A., de Carvalho, P. and Lopes, M.L. (2007) Doppler and Birth Weight Z Score: Predictors for Adverse Neonatal Outcome in Severe Fetal Compromise. Cardiovascular Ultrasound, 5, 15-22. https://doi.org/10.1186/1476-7120-5-15

[4] Gonsoulin, W. (1991) Umbilical Artery Doppler Waveform Analysis: A Randomized Study on Effect on Outcome. American Journal of Obstetrics and Gynecology, 164, 370. https://doi.org/10.1016/0002-9378(91)91191-X

[5] Alfirevic, Z. and Neilson, J.P. (1995) Doppler Ultrasonography in High-Risk Pregnancies: Systematic Review with Meta-Analysis. American Journal of Obstetrics and Gynecology, 172, 1379-1387. https://doi.org/10.1016/0002-9378(95)90466-2

[6] Figueras, F., Eixarch, E., Gratacos, E. and Gardosi, J. (2008) Predictiveness of Antenatal Umbilical Artery Doppler for Adverse Pregnancy Outcome in Small-forGestational-Age Babies According to Customised Birth Weight Centiles: Population-Based Study. British Journal of Obstetrics and Gynaecology, 115, 590-594. https://doi.org/10.1111/j.1471-0528.2008.01670.x

[7] Anshul, D., Neelu, S. and Suneeta, G. (2010) Significance of Umbilical Artery Doppler Velocimetry in the Perinatal Outcome of the Growth Restricted Fetuses. The Journal of Obstetrics and Gynecology of India, 60, 38-43. https://doi.org/10.1007/s13224-010-0007-2

[8] Valea, I., Tinto, H., Drabo, M.K., Huybregts, L., Sorgho, H., Ouedraogo, J.B., et al. (2012) An Analysis of Timing and Frequency of Malaria Infection during Pregnancy in Relation to the Risk of Low Birth Weight, Anaemia and Perinatal Mortality in Burkina Faso. Malaria Journal, 11, 71-78. https://doi.org/10.1186/1475-2875-11-71

[9] Wilson, N.O., Ceesay, F.K., Hibbert, J.M., Driss, A., Obed, S.A., Adjei, A.A., Gyasi, R.K., Anderson, W.A. and Stiles, J.K. (2012) Pregnancy Outcomes among Patients with Sickle Cell Disease at Korle-Bu Teaching Hospital, Accra, Ghana: Retrospective Cohort Study. The American Society of Tropical Medicine and Hygiene, 86, 936-942. https://doi.org/10.4269/ajtmh.2012.11-0625

[10] Morris, R.K., Malin, G., Robson, S.C., Kleijnen, J., Zamora, J., Khan, K.S.F., et al. (2011) Umbilical Artery Doppler to Predict Compromise of Fetal/Neonatal Wellbeing in a High-Risk Population: Systematic Review and Bivariate Meta-Analysis. Ultrasound in Obstetrics \& Gynecology, 37, 135-142.

https://doi.org/10.1002/uog.7767

[11] Lopez-Mendez, M.L., Martinez-Gaytan, V., Cortes-Flores, R., Ramos-Gonzalez, R.M., Ochoa-Torres, M.A., Garza-Veloz, I., et al. (2013) Doppler Ultrasound Evaluation in Preeclampsia. BMC Research Notes, 6, 477-483. https://doi.org/10.1186/1756-0500-6-477

[12] Jacobson, S.L., Imhof, R., Manning, N., Mannion, V., Little, D., Rey, E., et al. (1990) The Value of Doppler Assessment of the Uteroplacental Circulation in Predicting Pre-Eclampsia or Intra-Uterine Growth Retardation. American Journal of Obstetrics and Gynecology, 162, 110-114. https://doi.org/10.1016/0002-9378(90)90832-R

[13] Kwon, J.Y., Kwon, H.S., Kim, Y.H. and Park, Y.W. (2006) Abnormal Doppler Velocimetry Is Related to Adverse Perinatal Outcome for Borderline Amniotic Fluid Index during Third Trimester. Journal of Obstetrics and Gynaecology Research, 32, 545-549. https://doi.org/10.1111/j.1447-0756.2006.00459.x 
[14] Dorman, E.K., Shulman, C.E., Kingdom, J., Bulmer, J.N., Mwendwa, J., Peshu, N., et al. (2002) Impaired Uteroplacental Blood Flow in Pregnancies Complicated by Falciparum Malaria. Ultrasound in Obstetrics \& Gynecology, 19, 165-170. https://doi.org/10.1046/j.0960-7692.2001.00545.x

[15] McClure, E.M., Meshnick, S.R., Lazebnik, N., Mungai, P., King, C.L., Hudgens, M., et al. (2014) A Cohort Study of Plasmodium falciparum Malaria in Pregnancy and Associations with Uteroplacental Blood Flow and Fetal Anthropometrics in Kenya. International Journal of Gynecology \& Obstetrics, 126, 78-82.

https://doi.org/10.1016/j.ijgo.2014.01.016

[16] Ome-Kaius, M., Karl, S., Wangnapi, R.A., Bolnga, J.W., Mola, G., Walker, J., et al. (2017) Effects of Plasmodium falciparum Infection on Umbilical Artery Resistance and Intrafetal Blood Flow Distribution: A Doppler Ultrasound Study from Papua New Guinea. Malaria Journal, 16, 35. https://doi.org/10.1186/s12936-017-1689-Z

[17] Arbeille, P., Carles, G., Bousquet, F., Body, G. and Lansac, J. (1998) Fetal Cerebral and Umbilical Artery Blood Flow Changes during Pregnancy Complicated by Malaria. Journal of Ultrasound in Medicine, 17, 223-229. https://doi.org/10.7863/jum.1998.17.4.223

[18] Arbeille, P., Carles, G., Georgescu, M., Tobal, N., Herault, S., Bousquet, F., et al. (2003) Consequences of Reduced Umbilical and Increased Foetal Cerebral Flow during Malaria Crisis on Foetal Behaviour. Parasitology, 126, 513-519.

[19] Hwang, H.S., Kim, Y.H., Kwon, J.Y. and Park, Y.W. (2010) Uterine and Umbilical Artery Doppler Velocimetry as a Predictor for Adverse Pregnancy Outcomes in Pregnant Women with Anemia. Journal of Perinatal Medicine, 38, 467-471. https://doi.org/10.1515/jpm.2010.047

[20] Goffinet, F., Paris-Llado, J., Nisand, I. and Breart, G. (1997) Umbilical Artery Doppler Velocimetry in Unselected and Low Risk Pregnancies: A Review of Randomized Controlled Trials. British Journal of Obstetrics and Gynaecology, 104, 425-430. https://doi.org/10.1111/j.1471-0528.1997.tb11493.x

[21] Divon, M.Y. (1996) Umbilical Artery Doppler Velocimetry: Clinical Utility in High-Risk Pregnancies. American Journal of Obstetrics and Gynecology, 174, 10-14. https://doi.org/10.1016/S0002-9378(96)70365-4

[22] Carles, G., Tobal, N., Raynal, P., Herault, S., Beucher, G., Marret, H., et al. (2003) Doppler Assessment of the Fetal Cerebral Hemodynamic Response to Moderate or Severe Maternal Anemia. American Journal of Obstetrics and Gynecology, 188, 794-799. https://doi.org/10.1067/mob.2003.177

[23] Bricker, L. and Neilson, J.P. (2000) Routine Doppler Ultrasound in Pregnancy. Cochrane Database of Systematic Reviews, 2, CD001450. https://doi.org/10.1002/14651858.CD001450

[24] Westergaard, H.B., Langhoff-Roos, J., Lingman, G., Marsál, K. and Kreiner, S. (2001) A Critical Appraisal of the Use of Umbilical Artery Doppler Ultrasound in High-Risk Pregnancies: Use of Metaanalyses in Evidence-Based Obstetrics. Ultrasound in Obstetrics \& Gynecology, 17, 466-476.

https://doi.org/10.1046/j.1469-0705.2001.00415.x 